\title{
Decitabine versus best supportive care in older patients with refractory anemia with excess blasts in transformation (RAEBt) - results of a subgroup analysis of the randomized phase III study 06011 of the EORTC Leukemia Cooperative Group and German MDS Study Group (GMDSSG)
}

\author{
Heiko Becker ${ }^{1}$ - Stefan Suciu ${ }^{2}$ Björn Hans Rüter ${ }^{1}$ - Uwe Platzbecker ${ }^{3}$. \\ Aristoteles Giagounidis ${ }^{4}$ • Dominik Selleslag ${ }^{5}$ - Boris Labar ${ }^{6}$. Ulrich Germing ${ }^{7}$. \\ Helmut R. Salih ${ }^{8}$ - Petra Muus ${ }^{9} \cdot$ Karl-Heinz Pflüger $^{10}$ • Anne Hagemeijer ${ }^{11}$. \\ Hans-Eckart Schaefer ${ }^{12}$ • Valeria Fiaccadori ${ }^{2}$ • Frédéric Baron ${ }^{13}$ - Arnold Ganser ${ }^{14}$. \\ Carlo Aul $^{15}$. Theo de Witte ${ }^{9}$ Pierre W. Wijermans ${ }^{16}$ • Michael Lübbert ${ }^{1}$
}

\begin{abstract}
In the European Organisation for Research and Treatment of Cancer (EORTC)/GMDSSG phase III trial 06011, we compared decitabine $\left(15 \mathrm{mg} / \mathrm{m}^{2}\right.$ every $8 \mathrm{~h}$ for 3 days) with best supportive care (BSC) in patients $\geq 60$ years with myelodysplastic syndromes (MDS) by FrenchAmerican-British (FAB) criteria. Here, we reinvestigate trial 06011 for the activity and efficacy specifically in patients with refractory anemia with excess blasts in transformation (RAEBt). Response rates in the decitabine arm $(N=40)$ were
\end{abstract}

Electronic supplementary material The online version of this article (doi:10.1007/s00277-015-2489-6) contains supplementary material, which is available to authorized users.

Michael Lübbert

michael.luebbert@uniklinik-freiburg.de

1 Department of Medicine I, Medical Center-University of Freiburg, Freiburg, Germany

2 EORTC Headquarters, Brussels, Belgium

3 Department of Internal Medicine I, University Carl Gustav Carus, Dresden, Germany

4 Department of Hematology, Oncology and Palliative Care, Marienhospital Düsseldorf, Düsseldorf, Germany

5 Department of Hematology, AZ St-Jan, Brugge, Belgium

6 University Hospital Center Rebro, Zagreb, Croatia

7 Department of Hematology, Oncology and Clinical Immunology, University Hospital Düsseldorf, Düsseldorf, Germany as follows: complete or partial remission, $15 \%$; hematologic improvement, $15 \%$; resistant disease, $30 \%$. RAEBt patients in the decitabine arm had longer progression-free survival (PFS; hazard ratio (HR) $0.30,95 \%$ confidence interval (CI) 0.18-0.51; median, 6.2 vs 2.8 months) and overall survival (OS; HR 0.68, $95 \%$ CI 0.42-1.11; median, 8.0 vs 6.0 months) than in the BSC arm $(N=35)$. Censoring at allogeneic hematopoietic stem cell transplantation, the OS difference between the treatment groups increased, particularly among patients
8 Department of Hematology, Oncology and Immunology, Eberhard Karls University, Tübingen, Germany

9 Radboud University Medical Center, Nijmegen, Netherlands

10 Department of Medicine II, DIAKO Bremen, Bremen, Germany

11 Center for Human Genetics, University of Leuven, Leuven, Belgium

12 Institute of Pathology, University of Freiburg, Freiburg, Germany

13 Centre Hospitalier Universitaire Sart-Tilman, Liège, Belgium

14 Department of Hematology, Hemostasis, Oncology and Stem Cell Transplantation, Hannover Medical School, Hannover, Germany

15 Department of Hematology and Oncology, St. Johannes Hospital, Duisburg, Germany

16 Department of Internal Medicine, Haga Ziekenhuis, The Hague, Netherlands 
aged 60-74 years (HR 0.48, $95 \%$ CI $0.26-0.89$ ). After regrouping the study cohort according to World Health Organization (WHO) criteria, patients with acute myeloid leukemia (AML) (i.e., $\geq 20 \%$ blasts) in the decitabine arm $(N=27)$ also had longer PFS than in the BSC arm $(N=23)$ (HR $0.46,95 \%$ CI $0.26-0.83$; median, 6.2 vs 2.8 months). In conclusion, 3day decitabine displays clinical activity and efficacy in MDS and/or AML with 5-30 \% blood or 20-30\% marrow blasts.

Keywords RAEBt $\cdot$ MDS $\cdot$ AML $\cdot$ Leukemia $\cdot$ Allogeneic transplantation $\cdot$ Epigenetic therapy

\section{Introduction}

Treatment options in patients with myelodysplastic syndromes (MDS) and high blast counts are limited. The median overall survival (OS) of patients with refractory anemia with excess blasts in transformation (RAEBt) according to the French-American-British (FAB) classification is only approximately 6 months, if treated with best supportive care (BSC) $[1,2]$, but more intensive therapy is often not feasible due to older age, poor performance status, or comorbidities [3-5].

Fenaux et al. showed that treatment with the hypomethylating agent azacitidine prolongs OS among older patients with MDS by FAB criteria and $20-30 \%$ bone marrow (BM) blasts, compared to BSC [6]. A benefit by the hypomethylating drug decitabine in such patients has not yet been established. Among patients with acute myeloid leukemia (AML) according to World Health Organization (WHO) criteria and 20-30\% BM blasts, Kantarjian et al. observed no significant improvement of OS by decitabine given at the 5day schedule ( $20 \mathrm{mg} / \mathrm{m}^{2}$ every day for 5 days), compared with BSC or low-dose cytarabine [7].

In the phase III trial 06011, we compared the 3-day schedule of decitabine $\left(15 \mathrm{mg} / \mathrm{m}^{2}\right.$ every $8 \mathrm{~h}$ for 3 days) with BSC in patients $\geq 60$ years with MDS (FAB) not eligible for intensive therapy [8]. Decitabine administered at the 3-day schedule for MDS (FAB) is in line with its approval status in the USA.

In the present analysis, we examine trial 06011 for the activity and efficacy of decitabine specifically in patients with MDS (FAB) and higher blast counts (i.e., RAEBt) and in patients fulfilling the WHO criteria for AML. In both patient groups, we show an improved outcome in the decitabine compared with the BSC arm.

\section{Patients and methods}

\section{Patients}

Eligibility criteria of trial 06011 (clinicaltrials.gov Id NCT00043134) have been previously reported [8] and included the following: patients $\geq 60$ years old; primary or treatment-related MDS or chronic myelomonocytic leukemia (CMML) according to FAB criteria [9]; International Prognostic Scoring System (IPSS) intermediate-1, intermediate-2, or high risk [1]; BM blasts of 11 to $30 \%$, or $\leq 10 \%$ and poor cytogenetics according to IPSS criteria; Eastern Cooperative Oncology Group (ECOG) performance status of 0 to 2 ; and ineligible for intensive chemotherapy as judged by the treating physician. No previous treatment with standard chemotherapy or a hypomethylating agent was allowed. The study was performed in accordance with the Declaration of Helsinki and approved by the local ethics committees at all participating sites. All patients provided written informed consent.

\section{Study design}

Patients were randomly assigned (1:1) to receive either decitabine and BSC or BSC only. Crossover to the experimental arm was prohibited. Decitabine $\left(15 \mathrm{mg} / \mathrm{m}^{2}\right.$ in two doses over $2 \mathrm{~h}$ each) was given intravenously every $8 \mathrm{~h}$ for 3 days in 6 -week cycles [8]. If regeneration of hematologic parameters was insufficient, the interval between cycles could be extended up to 10 weeks. Reasons for exit from study were predefined as disease progression, transformation to AML (FAB), unacceptable toxicity, or per protocol (i.e., eight courses of decitabine or ten courses in case complete remission [CR] was achieved after eight courses).

\section{Endpoints}

Primary endpoint was OS (measured from the date of random assignment until death from any cause or last follow-up (censored observation)) [8]. Secondary endpoints were AML-free survival (AMLFS; measured from the date of random assignment to transformation to AML [FAB] or death from any cause, whichever occurred first), progression-free survival (PFS; measured from the date of random assignment to progression, relapse after achievement of CR or partial response [PR], or death, whichever occurred first), and best response rate. Responses were assessed according to International Working Group criteria for MDS defined by the FAB classification [10]. BM examinations were planned after every other cycle of decitabine and at weeks 24 and 48 in the BSC arm, or earlier in both arms if progression was suspected. Blood smears, BM aspirates and biopsies, and cytogenetics were centrally reviewed.

\section{Statistical analyses}

Survival estimates were calculated using the Kaplan-Meier method; standard errors of the estimates were obtained via 
the Greenwood formula [11]. The two-sided, log-rank test evaluated differences between survival distributions. The Cox proportional hazards model was used to obtain hazard ratio (HR) estimates and corresponding confidence intervals (CIs). Subgroup analyses were performed using Forest plot techniques [12]. For main efficacy analyses, the intent-totreat principle was followed. As sensitivity analysis for OS comparison, the follow-up was censored at time of allogeneic hematopoietic stem cell transplantation (alloHSCT). The database was located at the European Organisation for Research and Treatment of Cancer (EORTC) Headquarters, and all analyses were performed using SAS software (version 9.3 and 9.4, SAS Institute, Cary, NC, USA).

\section{Results}

\section{Characteristics of the patients with RAEBt}

From October 2002 through May 2007, 233 patients from 40 centers in nine European countries were randomly assigned to receive decitabine $(N=119)$ or BSC $(N=114)$ within trial 06011. Among the 233 patients, 75 patients were diagnosed with RAEBt and 125 with RAEB. The remaining 33 patients had refractory anemia (RA), refractory anemia with ring sideroblasts (RARS), CMML, or AML by FAB criteria.

Of the 75 RAEBt patients, 40 were randomized into the decitabine and 35 into the BSC arm (Fig. 1). The treatment arms were well balanced. The median age was similar in both treatment groups, i.e., 69.5 years in the decitabine and 69 years in the BSC arm. Patients in the decitabine arm tended to more often have WBC less than $2.5 \times 10^{9} / \mathrm{L}$ and good- or intermediate-risk cytogenetics according to IPSS criteria, but there were no differences for sex, ECOG performance status, or IPSS risk (Table 1).

With regard to the blast counts, 19 (47.5\%) of the RAEBt patients in the decitabine arm and $18(51.4 \%)$ of those in the BSC arm had $21 \%$ or more BM blasts. The remaining patients were classified as having RAEBt based on the presence of 5$30 \%$ blasts in the peripheral blood or of Auer rods.

The median number of decitabine courses administered to the RAEBt patients was 2.5 (mean, 3.9; range 0-10), which equals approximately 4 months of treatment. Two (5\%) patients did not receive the study drug, $10 \%$ received one course, $35 \%$ two courses, $27.5 \%$ three to six courses, and $22.5 \%$ eight or more courses.

\section{Outcome among the patients with RAEBt}

Among the RAEBt patients in the decitabine arm, $30 \%$ reached either CR (10\%), PR (5\%), or hematologic improvement (HI; $15 \%$ ); stable disease (SD) occurred in $30 \%$, and in another $30 \%$, the disease was primarily progressive. In the BSC arm, no patient achieved CR, PR, or HI; $14.3 \%$ of the patients experienced a period of $\mathrm{SD}$, and $80 \%$ directly went into PD (Table 2).

The favorable response rates of the RAEBt patients in the decitabine arm translated into a PFS that was highly significantly longer than that of patients under BSC $(P<0.001$; Table 2, Fig. 2a). The risk of PD, relapse, or death among RAEBt patients in the decitabine arm was only $30 \%$ of that of RAEBt patients under BSC (Table 2). RAEBt patients in the decitabine arm also had a significantly improved AMLFS, compared with patients in the BSC arm $(P=0.003)$ (Fig. 2b).

In addition to the significant improvements in PFS and AMLFS, RAEBt patients in the decitabine arm had a,
Fig. 1 CONSORT diagram considering the patients with RAEBt in trial 06011. A CONSORT diagram considering the entire cohort is provided elsewhere [8]

\begin{tabular}{|c|c|}
\hline \multicolumn{2}{|c|}{ Assessed for eligibility, $n=75$} \\
\hline \multicolumn{2}{|c|}{ Randomly allocated, n=75 } \\
\hline $\begin{array}{l}\text { Allocated to best supportive care, } n=35 \\
\text { Started allocated intervention, } n=35 \\
\text { Did not receive allocated intervention, } n=0\end{array}$ & $\begin{array}{l}\text { Allocated to decitabine, } n=40 \\
\text { Started allocated intervention, } n=38 \\
\text { Did not receive allocated intervention, } n=2\end{array}$ \\
\hline $\begin{array}{l}\text { Lost to follow-up, } n=0 \\
\text { Normal completion of study protocol, } n=0 \\
\text { Discontinued intervention, } n=35 \\
\text { Progressive disease, } n=18 \\
\text { Toxicity, } n=0 \\
\text { Hypoplasia, } n=0 \\
\text { Death, } n=9 \\
\text { Refusal of the patient, } n=4 \\
\text { Protocol violation, } n=2 \\
\text { Ineligibility, } n=0 \\
\text { Other, } n=2\end{array}$ & $\begin{array}{l}\text { Lost to follow-up, } \mathrm{n}=0 \\
\text { Normal completion of study protocol, } \mathrm{n}=11 \\
\text { Discontinued intervention, } \mathrm{n}=29 \\
\text { Progressive disease, } \mathrm{n}=15 \\
\text { Toxicity, } \mathrm{n}=4 \\
\text { Hypoplasia, } \mathrm{n}=0 \\
\text { Death, } \mathrm{n}=5 \\
\text { Refusal of the patient, } \mathrm{n}=2 \\
\text { Protocol violation, } \mathrm{n}=2 \\
\text { Ineligibility, } \mathrm{n}=0 \\
\text { Other, } \mathrm{n}=1\end{array}$ \\
\hline
\end{tabular}


Table 1 Pretreatment features of patients with RAEBt

\begin{tabular}{|c|c|c|}
\hline \multirow[t]{2}{*}{ Feature } & \multicolumn{2}{|l|}{ RAEBt } \\
\hline & Decitabine $(N=40)$ & $\operatorname{BSC}(N=35$ \\
\hline \multicolumn{3}{|l|}{ Age (years) } \\
\hline Median & 69.5 & 69 \\
\hline Range & $61-90$ & $61-80$ \\
\hline \multicolumn{3}{|l|}{ Age (years), $N(\%)$} \\
\hline $60-64$ & $10(25.0)$ & $6(17.1)$ \\
\hline $65-69$ & $10(25.0)$ & $12(34.3)$ \\
\hline $70-74$ & $8(20.0)$ & $10(28.6)$ \\
\hline$\geq 75$ & $12(30.0)$ & $7(20.0)$ \\
\hline \multicolumn{3}{|l|}{ Sex, $N(\%)$} \\
\hline Male & $29(72.5)$ & $24(68.6)$ \\
\hline Female & $11(27.5)$ & $11(31.4)$ \\
\hline \multicolumn{3}{|c|}{ ECOG performance status, $N(\%)$} \\
\hline 0 & $8(20.0)$ & $10(28.6)$ \\
\hline 1 & $29(72.5)$ & $19(54.3)$ \\
\hline 2 & $3(7.5)$ & $6(17.1)$ \\
\hline \multicolumn{3}{|l|}{$\mathrm{WBC}, \times 10^{9} / \mathrm{L}$} \\
\hline$<2.5$ & $20(50.0)$ & $11(31.4)$ \\
\hline $2.5-<10$ & $11(27.5)$ & $18(51.4)$ \\
\hline$\geq 10$ & $8(20.0)$ & $6(17.1)$ \\
\hline \multicolumn{3}{|l|}{$\%$ BM blasts, $N(\%)$} \\
\hline$\leq 20 \%$ & $21(52.5)$ & $17(18.6)$ \\
\hline $21-30 \%{ }^{\mathrm{a}}$ & $19(47.5)$ & $18(51.4)$ \\
\hline \multicolumn{3}{|l|}{ Disease status } \\
\hline Primary, untreated & $8(20.0)$ & $1(2.9)$ \\
\hline Primary, pretreated ${ }^{\mathrm{b}}$ & $28(70.0)$ & $31(88.6)$ \\
\hline Secondary & $4(10.0)$ & $3(8.6)$ \\
\hline \multicolumn{3}{|c|}{ Cytogenetic risk (IPSS), $N(\%)$} \\
\hline Good & $16(40.0)$ & $5(14.3)$ \\
\hline Intermediate & $4(10.0)$ & $5(14.3)$ \\
\hline Poor & $14(35.0)$ & $17(48.6)$ \\
\hline Failed & $5(12.5)$ & $8(22.9)$ \\
\hline Not assessed & $1(2.5)$ & $0(0.0)$ \\
\hline \multicolumn{3}{|l|}{ IPSS, $N(\%)$} \\
\hline Intermediate 1 & $2(5.0)$ & $0(0.0)$ \\
\hline Intermediate 2 & $12(30.0)$ & $13(37.1)$ \\
\hline High risk & $26(65.0)$ & $22(62.9)$ \\
\hline \multicolumn{3}{|c|}{ Time from diagnosis to random assignment (months), $N(\%)$} \\
\hline$\geq 3$ & $16(40.0)$ & $20(57.1)$ \\
\hline$<3$ & $24(60.0)$ & $15(42.9)$ \\
\hline
\end{tabular}

$R A E B t$ refractory anemia with excess blasts in transformation, $B S C$ best supportive care, $B M$ bone marrow, ECOG Eastern Cooperative Oncology Group, IPSS International Prognostic Scoring System

${ }^{a}$ Includes one patient with $40 \%$ BM blasts

${ }^{\mathrm{b}}$ For example, with transfusions, hydroxyurea, growth factors, immunosuppressive therapy although not significant $(P=0.12)$, prolongation of their median OS ( 8.0 vs 6.0 months) (Table 2, Fig. 2c). As post-progression treatment, particularly in the form of potentially curative alloHSCT, may influence OS, we censored the survival of the two patients in the decitabine and five patients in the BSC arm, who received an alloHSCT, at the time of HSCT. We observed that the difference in the OS between the two treatment groups slightly increased in favor of those RAEBt patients treated in the decitabine arm $(P=0.07)$ (Table 2, Fig. 3a).

All RAEBt patients who received an alloHSCT were 60 74 years old. We restricted further exploratory OS analyses to this age group, i.e., to patients who, as defined by age, are potential candidates for an alloHSCT following reduced intensity conditioning. Within this age group and censored at the time of alloHSCT, RAEBt patients in the decitabine arm had longer OS than those in the BSC arm $(P=0.018)$ (Table 2, Fig. 3b).

\section{Outcome comparisons between patients with RAEBt vs RAEB}

In our initial report on the entire study cohort of trial 06011, we observed a significantly longer PFS for patients in the decitabine arm, but no significant differences regarding AMLFS or OS between the treatment arms [8]. Thus, we wondered how the results of the present analyses in RAEBt patients compared to the results in other FAB subgroups. Since patient numbers were too small for conclusive analyses in RA ( $N=5$ in the decitabine arm, $N=8$ in the BSC arm), RARS $(N=3, N=2)$, and CMML $(N=10, N=4)$, we focused on the treatment comparison in RAEBt and RAEB patients (Fig. 4a-c). We observed that the effect of decitabine on both PFS and AMLFS was significantly greater among the RAEBt than RAEB patients (tests for heterogeneity, $P=0.0001$ and $P=0.004$, respectively). With regard to OS, the impact of decitabine vs BSC was also stronger in the RAEBt than the RAEB patients, although the difference did not reach statistical significance (test for heterogeneity, $P=$ $0.07)$.

\section{Survival in subgroups of RAEBt patients}

We further explored whether the positive impact of decitabine can be observed in subgroups of RAEBt patients defined by age, BM blasts, IPSS risk, or time from diagnosis (Fig. 5, Supplementary Fig. S1). With regard to PFS, AMLFS, and OS, there were no significant differences in the effect of decitabine among the various subgroups. 
Table 2 Treatment response of patients with RAEBt

\begin{tabular}{|c|c|c|c|c|}
\hline \multirow[t]{2}{*}{ Outcome endpoint } & \multicolumn{4}{|l|}{ RAEBt } \\
\hline & Decitabine $(N=40)$ & $\operatorname{BSC}(N=35)$ & $\mathrm{HR}(95 \% \mathrm{CI})$ & $P$ value $^{\mathrm{a}}$ \\
\hline \multicolumn{5}{|l|}{ Best response, $N(\%)$} \\
\hline Complete remission & $4(10.0)$ & $0(0.0)$ & \multirow[t]{7}{*}{ n.a. } & \multirow[t]{7}{*}{ n.a. } \\
\hline Partial remission & $2(5.0)$ & $0(0.0)$ & & \\
\hline Hematologic improvement & $6(15.0)$ & $0(0.0)$ & & \\
\hline Stable disease & $12(30.0)$ & $5(14.3)$ & & \\
\hline Progressive disease & $12(30.0)$ & $28(80.0)$ & & \\
\hline Hypoplasia & $2(5.0)$ & $0(0.0)$ & & \\
\hline Not evaluable & $2(5.0)$ & $2(5.7)$ & & \\
\hline \multicolumn{5}{|l|}{ Progression-free survival } \\
\hline Median (months) & 6.2 & 2.8 & \multirow[t]{2}{*}{$0.30(0.18-0.51)$} & \multirow[t]{2}{*}{$<0.001$} \\
\hline$\%$ at 6 months & 50.0 & 20.0 & & \\
\hline \multicolumn{5}{|l|}{ AML (FAB)-free survival } \\
\hline Median (months) & 7.0 & 3.7 & \multirow[t]{2}{*}{$0.49(0.30-0.80)$} & \multirow[t]{2}{*}{0.003} \\
\hline$\%$ at 6 months & 52.5 & 31.4 & & \\
\hline \multicolumn{5}{|l|}{ Overall survival } \\
\hline Median (months) & 8.0 & 6.0 & \multirow[t]{2}{*}{$0.68(0.42-1.11)$} & \multirow[t]{2}{*}{0.12} \\
\hline$\%$ at 6 months & 65.0 & 51.4 & & \\
\hline \multicolumn{5}{|c|}{ Overall survival, censored at time of alloHSCT } \\
\hline Median (months) & 8.0 & 5.9 & \multirow[t]{2}{*}{$0.63(0.38-1.04)$} & \multirow[t]{2}{*}{0.07} \\
\hline$\%$ at 6 months & 66.7 & 48.1 & & \\
\hline \multicolumn{5}{|c|}{ Overall survival, censored at time of alloHSCT, 60-74 years ${ }^{\mathrm{b}}$} \\
\hline Median (months) & 8.0 & 5.8 & \multirow[t]{2}{*}{$0.48(0.26-0.89)$} & \multirow[t]{2}{*}{0.018} \\
\hline$\%$ at 6 months & 63.1 & 41.2 & & \\
\hline
\end{tabular}

$R A E B t$ refractory anemia with excess blasts in transformation, $B S C$ best supportive care, $H R$ hazard ratio, $C I$ confidence interval, n.a. not applicable, $A M L$ acute myeloid leukemia, FAB French American British classification, alloHSCT allogeneic hematopoietic stem cell transplantation,

${ }^{\text {a }}$ Two-tailed log-rank test

${ }^{\mathrm{b}}$ Subgroup of patients aged 60-74 years: decitabine arm, $N=28$; BSC arm, $N=28$

\section{Characteristics and outcome of patients with AML according to WHO}

Patients with 20-30\% blasts in blood or BM have RAEB or RAEBt according to the FAB classification, but AML according to the WHO $[9,13]$. As the FAB classification is widely replaced by the $\mathrm{WHO}$ classification, we also investigated trial 06011 after regrouping the patients according to WHO criteria.

We identified 50 patients with AML and 20-30\% blasts in BM $(N=49)$ or blood $(N=1)$. Patients not diagnosed with AML and 20-30\% blasts were combined into the group of patients with MDS according to WHO $(N=164)$ or were those with CMML, BM blasts $>30 \%$, or no blast counts available. Of the AML patients, 27 were in the decitabine and 23 in the BSC arm (Supplementary Fig. S2). The median age was 70 years in both arms, and there were no major differences for sex, ECOG performance status, WBC, FAB subtype, cytogenetic risk, IPSS risk, and time from diagnosis to treatment assignment (Supplementary Table S1). A median of three courses was administered in the decitabine arm (mean 3.9, range $0-8) ; 37 \%$ of the patients received one to two courses, $18.5 \%$ each three courses or four to six courses, and $22.2 \%$ received all eight courses of the study protocol.

In the decitabine arm, $33.3 \%$ of the AML patients achieved CR (11.1\%), PR (11.1\%), or HI (11.1\%), and $37 \%$ progressed directly; in the BSC arm, no patient achieved CR, PR, or HI, and $73.9 \%$ of the patients had PD (Supplementary Table S2). AML patients in the decitabine arm had a significantly longer PFS than those in the BSC arm $(P=0.008$; Supplementary Table S2, Supplementary Fig. S3). However, this did not translate into a significantly improved OS of the patients in the decitabine arm, although the median OS was 9.8 months, compared to 5.9 months in patients receiving BSC only (Supplementary Table S2, Supplementary Fig. S3). When censored at the time of alloHSCT, the OS difference also did not reach statistical significance (Supplementary Table S2). 
Fig. 2 a Progression-free survival, b AML (FAB)-free survival, and $\mathbf{c}$ overall survival of RAEBt patients treated with decitabine or best supportive care (BSC). Further details are provided in Table 2

\section{a}

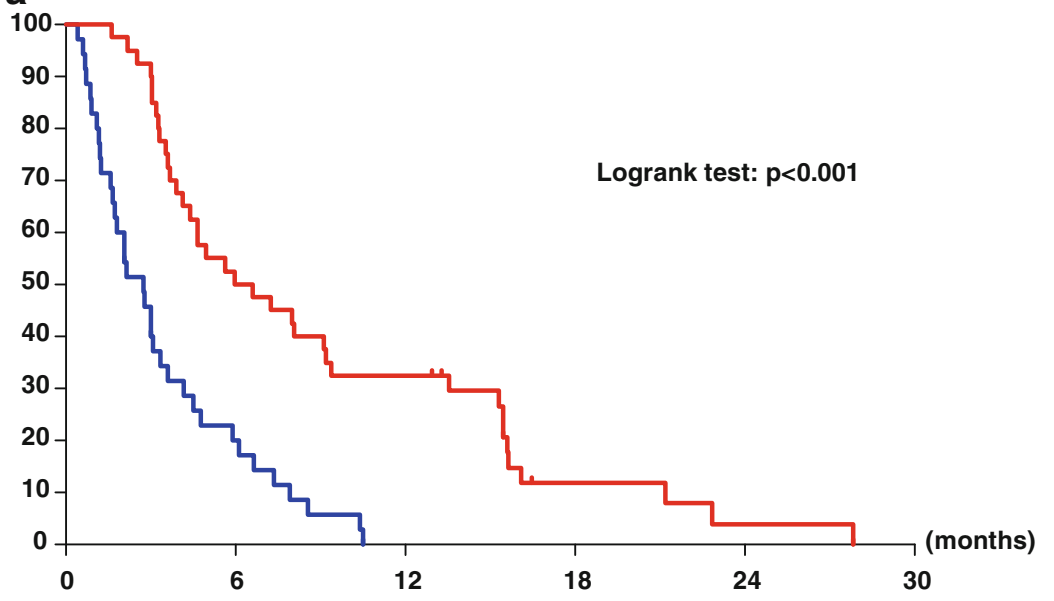

$\frac{\mathrm{O}}{35} \frac{\mathrm{N}}{35}$

$37 \quad 40$

Number of patients at risk :

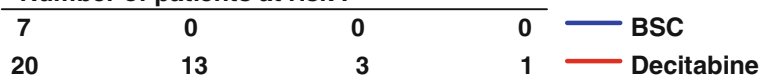

b

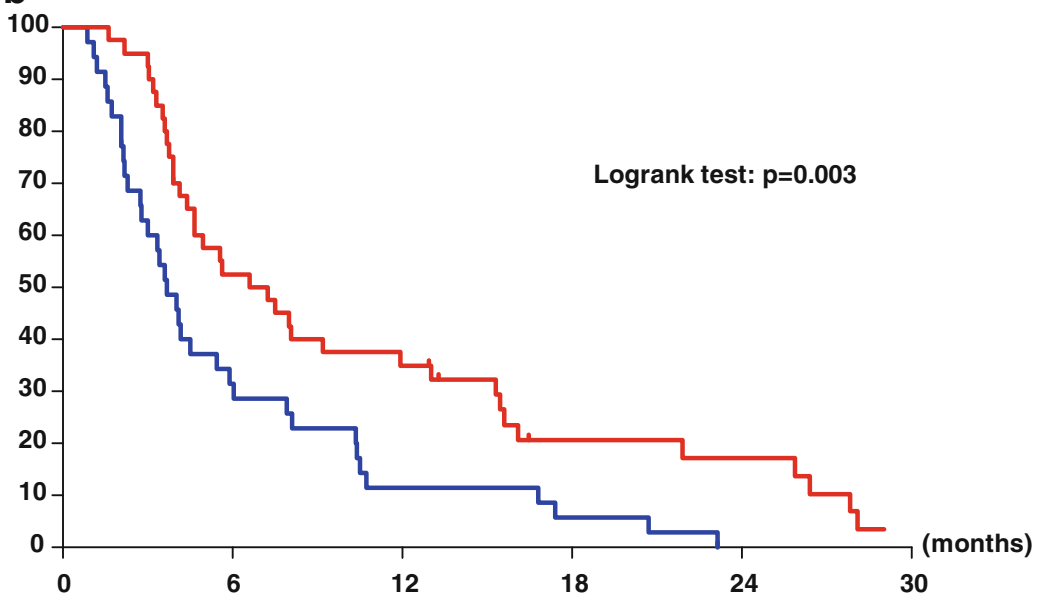

O N Number of patients at risk:

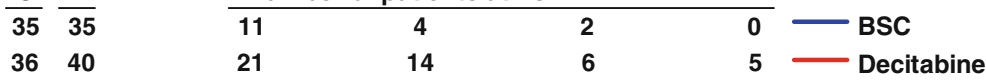

C

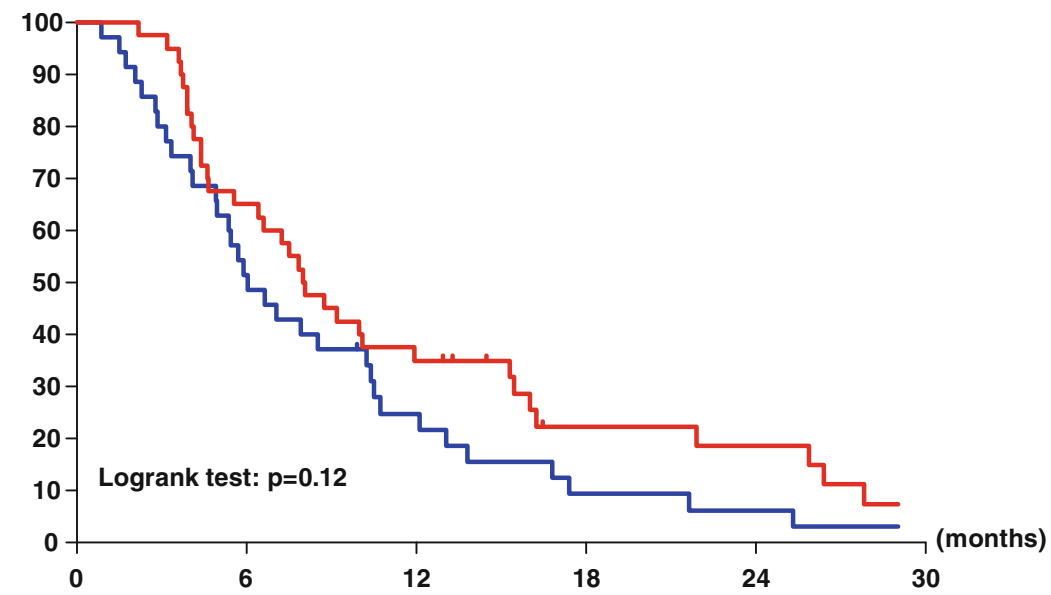

$0 \quad \mathrm{~N} \quad$ Number of patients at risk :

$\frac{\mathrm{O}}{33} \overline{\mathrm{N}}$

$34 \quad 40$
8

14

\section{3}

2 BSC

5 Decitabine 


\section{Discussion}

In patients with MDS and high blast counts, if ineligible for intensive therapy, decitabine and azacitidine have become standard of care. Fenaux et al. observed that older patients with MDS (FAB) and 20-30\% BM blasts who received azacitidine had longer OS than those receiving BSC only [6]. Such an effect of azacitidine had been also suggested in a previous pooled analysis of various studies [14]. A comparable effect of decitabine in this patient group has not yet been established.

Decitabine given at the 5-day schedule is approved in Europe for use in AML (WHO) in patients $\geq 65$ years not eligible for standard chemotherapy. In these patients, 5-day decitabine resulted in trends for a longer OS than treatment with BSC only or, more often, combined with low-dose cytarabine [7]. In subgroup analyses, no significant impact of decitabine on OS among patients with 20-30\% BM blasts was observed, although the median OS was prolonged by approximately 2 months in the decitabine arm (8.0 vs 6.1 months); no data on other survival endpoints were presented [7].

Decitabine at the 3-day schedule (in addition to the 5-day schedule) is approved in the USA for the treatment of MDS of all FAB subtypes, with a recommended duration of $3 \mathrm{~h}$ per infusion instead of $4 \mathrm{~h}$ as in our study. Kantarjian et al. observed that patients with MDS (FAB) receiving 3-day decitabine had a, by trend, longer AMLFS than with BSC [15]. In post hoc analyses, data from this study were combined
Fig. 3 Overall survival censored at the time of allogeneic hematopoietic stem cell transplantation of RAEBt patients treated with decitabine or best supportive care (BSC) considering a the entire cohort or b patients aged $60-74$ years. Further details are provided in Table 2 a

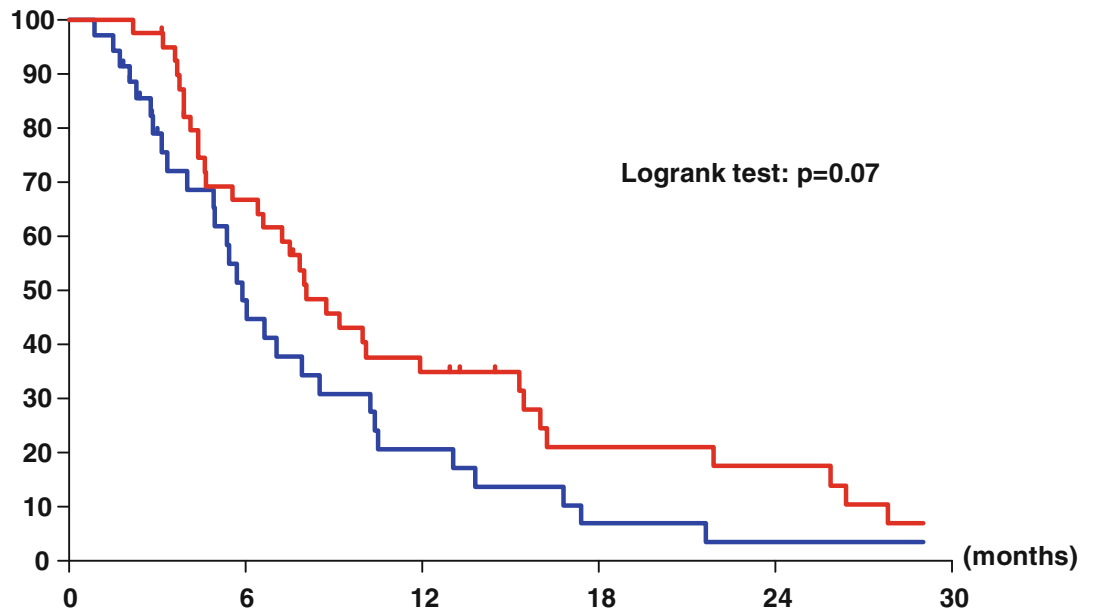

b

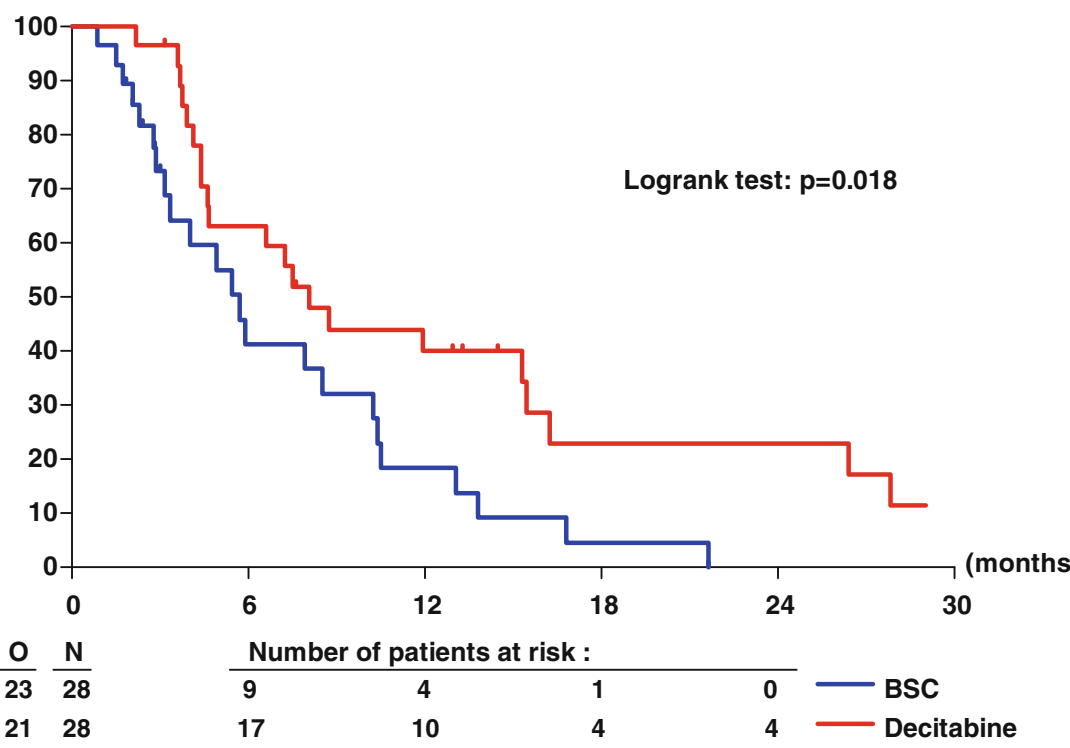


Fig. 4 Forest plots displaying a progression-free survival, b AML (FAB)-free survival, and $\mathbf{c}$ overall survival of patients with RAEBt or RAEB. $D A C$ decitabine, $B S C$ best supportive care, $O-E$ observed minus expected number of events, Var variance, $H R$ hazard ratio, $C I$ confidence interval, $F A B$ French-AmericanBritish classification assessed by the study coordinators (SC) a

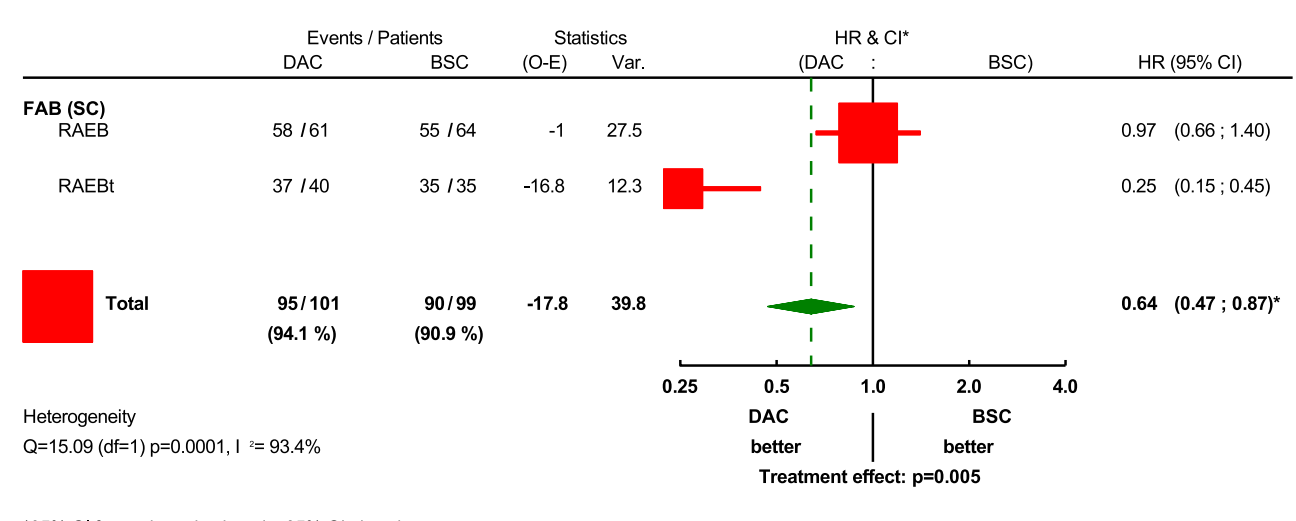

b

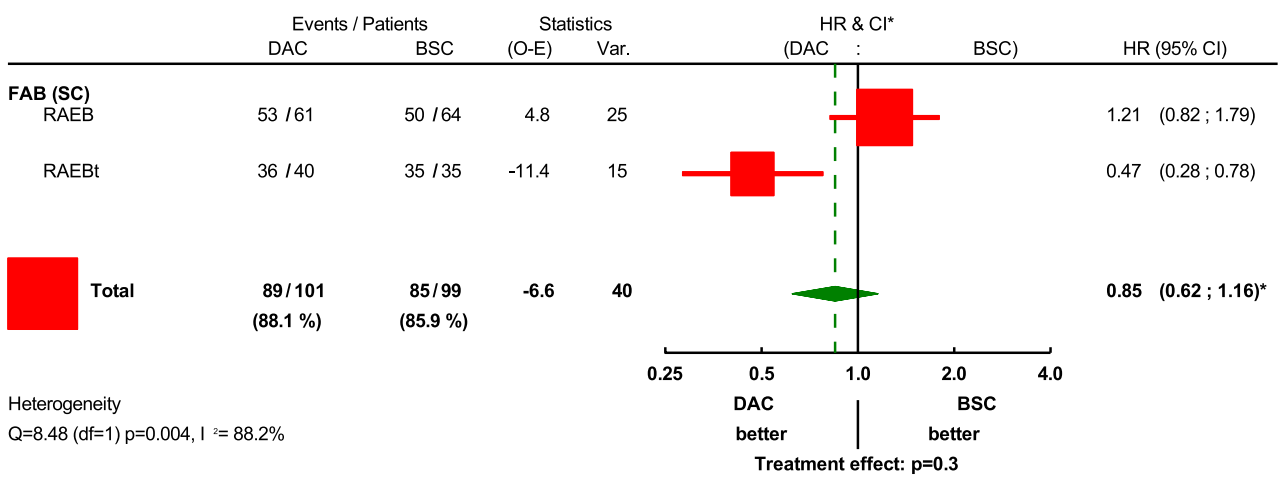

${ }^{*} 95 \% \mathrm{Cl}$ for totals and subtotals, $95 \% \mathrm{Cl}$ elsewhere

C

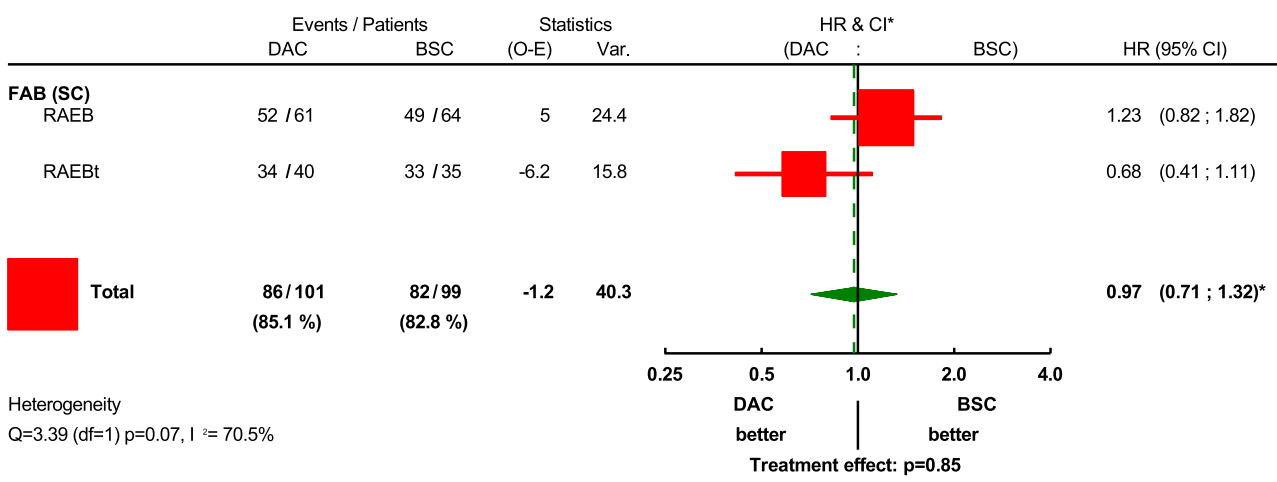

* $95 \% \mathrm{Cl}$ for totals and subtotals, $95 \% \mathrm{Cl}$ elsewhere with those of another trial, in which MDS (FAB) patients received 5-day decitabine $[16,17]$. Here, decitabine did not prolong the OS of patients with BM blasts $\geq 20 \%$ ( $89 \%$ had $20-30 \%$ BM blasts) to that of patients with MDS and less BM blasts [17].
In our phase III trial 06011, patients with MDS (FAB) were randomized to decitabine at the 3-day schedule or BSC only. As previously published [8], in the entire cohort, patients in the decitabine arm had longer PFS but similar OS compared with those in the BSC arm. For the present analysis, trial 
Fig. 5 Forest plot displaying progression-free survival of patients with RAEBt. The following factors were analyzed: patient age, bone marrow (BM) blasts, risk according to International Prognostic Scoring System (IPSS), and time from diagnosis to treatment assignment. $D A C$ decitabine, $B S C$ best supportive care, $O-E$ observed minus expected number of events, Var variance, $H R$ hazard ratio, $C I$ confidence interval, INT-1 intermediate-1 risk, INT-2 intermediate-2 risk, $H R$ high risk

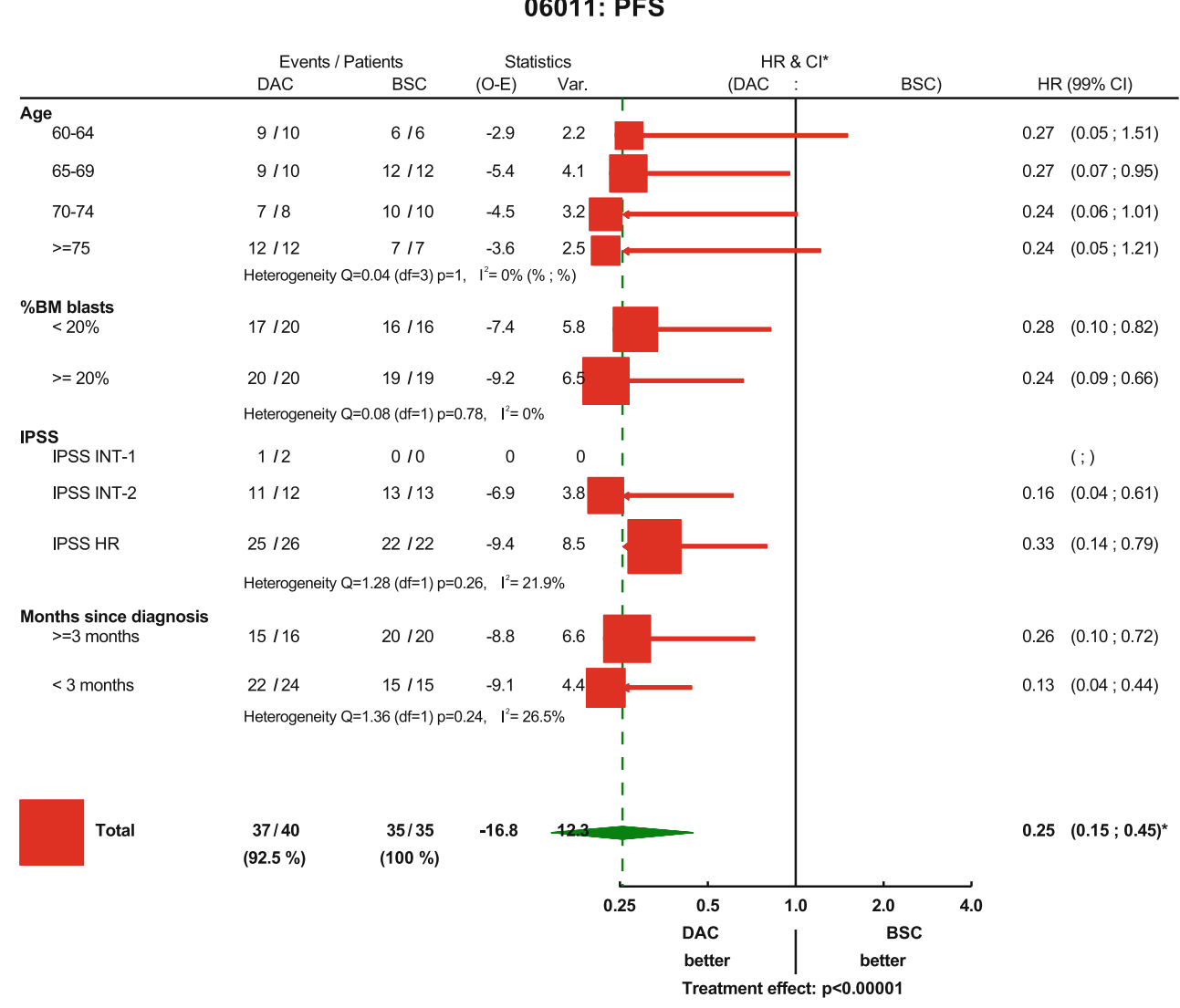

${ }^{*} 95 \% \mathrm{Cl}$ for totals and subtotals, $99 \% \mathrm{Cl}$ elsewhere
06011 gave us the opportunity to evaluate decitabine specifically among patients with RAEBt or AML (WHO) with low blast counts. Although the cohorts of RAEBt and AML (WHO) patients overlap, we decided to investigate both groups since the original study population was defined by FAB criteria, but the WHO classification is the one more widely used nowadays.

Among the RAEBt patients, those in the decitabine arm had, as expected, more favorable responses than those in the BSC arm. This is probably best reflected by the rates of patients whose disease was primarily progressive, which were $30 \%$ in the decitabine and $80 \%$ in the BSC arm. Accordingly, we observed a highly significant prolongation of PFS and AMLFS in the decitabine arm, and patients in the decitabine arm also tended to have a longer OS (median, $8.0 \mathrm{vs}$ 6.0 months). While the median OS in our study appears short in comparison with that reported by Fenaux et al. from the comparison of azacitidine and BSC only (median, 19.1 vs 13.4 months), the studies are not directly comparable due to differences in the patient cohorts, as displayed by the OS of patients in the BSC arms [6]. The few retrospective studies that compared decitabine and azacitidine did not specifically assess the survival among patients with RAEBt and/or 20$30 \%$ blasts [18-22]. Importantly, we also observed that the favorable impact of decitabine on the outcome of RAEBt patients was similar among various patient subgroups and significantly differed from that among RAEB patients.

Observing the strong impact of decitabine on PFS and AMLFS compared with the weaker effect on OS, we wondered whether this could be due to the treatment that patients received after being off-study. Among such treatments, an alloHSCT has the potential to cure from the disease and prolong survival. Indeed, when censoring at the time of alloHSCT, the benefit of decitabine on OS among RAEBt patients became more evident. This was particularly true for patients aged 60-74 years, thus for patients who are potential candidates for alloHSCT after reduced intensity conditioning.

When we regrouped the patients according to WHO criteria, patients with AML also had a longer PFS in the decitabine than in the BSC arm. However, in accordance to the observations in patients with 20-30\% BM blasts using 5day decitabine [7], the difference in OS between the decitabine and BSC arms was not significant.

The effect of decitabine on the survival endpoints in patients with AML (WHO) appeared weaker than in RAEBt patients. This difference may be explained by the following: Approximately half of the RAEBt patients were not included in the AML (WHO) cohort, as they did not have blast counts of 20-30\%; several RAEB patients were included in the AML (WHO) cohort, and decitabine had no impact on the 
survival endpoints among RAEB patients; the cohort of patients with AML (WHO) was relatively small.

Despite our result of a strong and favorable impact of 3-day decitabine in RAEBt rather than AML (WHO) with low blast counts, it would probably be misleading to conclude that 3day decitabine is solely effective in patients who strictly fulfill the FAB criteria of RAEBt, as opposed to those who have AML according to the WHO classification, considering the overlapping clinical and biologic features. It remains, however, elusive to what extent our findings can be transferred to patients with AML and $>30 \%$ blasts, particularly since the AML cases in our analyses are probably best represented by the WHO category of AML with myelodysplasia-related changes [23].

In patients $\geq 65$ years with AML and $>30 \%$ BM blasts (32\% of patients had AML with myelodysplasia-related changes), Dombret et al. compared azacitidine vs conventional care. They observed that patients in the azacitidine arm had longer OS than those in the BSC arm (median, 5.8 vs 3.7 months) [24]. In the previously mentioned study by Kantarjian et al., patients $\geq 65$ years with AML and $>30 \%$ BM blasts receiving 5-day decitabine also had a significantly longer OS than those receiving low-dose cytarabine ( $88 \%$ of patients) or BSC (12\%) (median, 7.1 vs 4.3 months) [7].

Considering the effect of decitabine among RAEBt patients in our study, it would have been of interest to know whether this correlates with the presence of certain genetic or epigenetic features of RAEBt and whether such features could predict the response to decitabine. Studies have suggested associations between the response to decitabine and genetic aberrations (e.g., monosomal karyotype, TET2 mutations), mRNA or microRNA expression, or DNA methylation [25-32]. Unfortunately, we did not have sufficient material to investigate molecular markers in our cohort. However, we are studying the predictive value of a monosomal karyotype for decitabine treatment in MDS (manuscript in preparation).

It remains to be established whether decitabine given at the 5-day schedule in RAEBt would resemble our results using the 3-day schedule. Due to the three infusions per day, the 3day schedule is not feasible in the outpatient setting. However, it is a valid option for patients hospitalized for or during treatment (e.g., due to poor performance status or long distance to the treatment center), and the more continuous application of decitabine in the 3-day schedule is supported by its relatively short plasma half-life [33].

In addition to the potential prolongation of OS, the positive effect on PFS and AMLFS among RAEBt patients may be used as bridge to alloHSCT, as the patient's general condition may improve under therapy. In older patients with AML, who are eligible for intensive chemotherapy, we currently perform a prospective trial of alloHSCT following the intensified 10day schedule of decitabine or a standard chemotherapy regimen (ClinicalTrials.gov Id NCT02172872).
Since the number of decitabine cycles was restricted to a maximum of 8 (or 10 in case that the patient achieved CR) in our study, we could not examine how a prolonged treatment with decitabine would have affected the survival endpoints. However, current data suggest that the hypomethylating therapy should be continued after achievement of a sustained response [34]. Maintenance therapy using hypomethylating agents may also be particularly effective in the postalloHSCT setting $[35,36]$.

In conclusion, our data point to the clinically relevant efficacy of decitabine using the 3-day schedule in patients with RAEBt and AML (WHO) with low blast counts, particularly by delaying progression or relapse, and, in case of RAEBt, also death.

Acknowledgments We thank the following additional EORTC Headquarters staff who worked on this study: Christine de Balincourt, Edith Bastiaens, Caroline Gilotay, Ann Marinus, Liv Meert, Thomas Vandenberghe, and Christine Waterkeyn.

Funding The study was supported by an educational grant from MGI Pharma and Johnson \& Johnson. The publication was supported by the EORTC Cancer Research Fund.

Conflict of interest Personal financial interests: none. Honoraria: U.P., Celgene, Novartis, Amgen; A.G., Celgene; D.S., Celgene, Novartis, Amgen; B.L., Novartis, MSD; U.G., MDS Register Düsseldorf, Johnson \& Johnson; H.R.S., Celgene, Novartis; A.G., Genzyme. Funding: U.P., Celgene, Novartis; U.G., MDS Register Düsseldorf; A.G., Novartis. M.L., Janssen-Cilag, Celgene, Teva. Employment: B.H.R., BöhringerIngelheim. Patent rights: none. Consultancy Work: U.P., Celgene, Amgen, Novartis; A.G., Celgene; D.S., Celgene, Novartis, Amgen; H.R.S., Novartis, Amgen, BMS; and A.G., Celgene, Novartis.

Author contribution Conception and design: H.B., S.S., A.Gi., P.M., A.Ga., C.A., T.d.W., and P.W.W.; provision of study materials or patients: B.H.R., U.P., A.Gi., D.S., B.L., U.G., H.R.S., P.M., K.-H.P., A.Ga., C.A., T.d.W., P.W.W., and M.L.; collection and assembly of data: S.S., B.H.R., U.P., A.Gi., D.S., U.G., P.M., K.-H.P., A.H., and M.L.; data analysis and interpretation: H.B., S.S., B.H.R., P.M., A.H., H.-E.S., V.F., F.B., T.d.W., P.W.W., and M.L.; drafting of the manuscript: H.B., S.S., and M.L.; and critical review and final approval of the manuscript: H.B., S.S., B.H.R., U.P., A.Gi., D.S., B.L., U.G., H.S., P.M., K.-H.P., A.H., H.-E.S., V.F., F.B., A.Ga., C.A., T.d.W., P.W.W., and M.L.

\section{References}

1. Greenberg P, Cox C, LeBeau MM et al (1997) International scoring system for evaluating prognosis in myelodysplastic syndromes. Blood 89:2079-2088

2. Germing U, Gattermann N, Strupp C, Aivado M, Aul C (2000) Validation of the WHO proposals for a new classification of primary myelodysplastic syndromes: a retrospective analysis of 1600 patients. Leuk Res 24:983-992

3. Kantarjian H, Beran M, Cortes J et al (2006) Long-term follow-up results of the combination of topotecan and cytarabine and other intensive chemotherapy regimens in myelodysplastic syndrome. Cancer 106:1099-1109

4. Deschler B, de Witte T, Mertelsmann R, Lübbert M (2006) Treatment decision-making for older patients with high-risk 
myelodysplastic syndrome or acute myeloid leukemia: problems and approaches. Haematologica 91:1513-1522

5. Estey EH (2013) Epigenetics in clinical practice: the examples of azacitidine and decitabine in myelodysplasia and acute myeloid leukemia. Leukemia 27:1803-1812

6. Fenaux P, Mufti GJ, Hellström-Lindberg E et al (2010) Azacitidine prolongs overall survival compared with conventional care regimens in elderly patients with low BM blast count acute myeloid leukemia. J Clin Oncol 28:562-569

7. Kantarjian HM, Thomas XG, Dmoszynska A et al (2012) Multicenter, randomized, open-label, phase III trial of decitabine versus patient choice, with physician advice, of either supportive care or low-dose cytarabine for the treatment of older patients with newly diagnosed acute myeloid leukemia. J Clin Oncol 30:2670 2677

8. Lübbert M, Suciu S, Baila L et al (2011) Low-dose decitabine versus best supportive care in elderly patients with intermediateor high-risk myelodysplastic syndrome (MDS) ineligible for intensive chemotherapy: final results of the randomized phase III study of the European Organisation for Research and Treatment of Cancer Leukemia Group and the German MDS Study Group. J Clin Oncol 29:1987-1996

9. Bennett JM, Catovsky D, Daniel MT et al (1982) Proposals for the classification of the myelodysplastic syndromes. Br J Haematol 51: 189-199

10. Cheson BD, Bennett JM, Kantarjian H et al (2000) World Health Organization (WHO) international working group. Report of an international working group to standardize response criteria for myelodysplastic syndromes. Blood 96:3671-3674

11. Kalbfleisch JD, Prentice RL (2002) The survival analysis of failure time data, 3rd edn. Wiley Inter-Science, Hoboken

12. Early Breast Cancer Trialists' Collaborative Group (1992) Systemic treatment of early breast cancer by hormonal, cytotoxic, or immune therapy: 133 randomised trials involving 31,000 recurrences and 24,000 deaths among 75,000 women. Lancet 339:71-85

13. Vardiman JW, Brunning RD, Arber DA et al (2008) Introduction and overview of the classification of the myeloid neoplasms. In: Swerdlow SH, Campo E, Harris NL et al (eds) WHO classification of tumours of haematopoietic and lymphoid tissues, 4th edn. IARC, Lyon, pp 18-30

14. Silverman LR, McKenzie DR, Peterson BL et al (2006) Further analysis of trials with azacitidine in patients with myelodysplastic syndrome: studies 8421,8921 , and 9221 by the Cancer and Leukemia Group B. J Clin Oncol 24:3895-3903

15. Kantarjian H, Issa JP, Rosenfeld CS et al (2006) Decitabine improves patient outcomes in myelodysplastic syndromes: results of a phase III randomized study. Cancer 106:1794-1803

16. Steensma DP, Baer MR, Slack JL et al (2009) Multicenter study of decitabine administered daily for 5 days every 4 weeks to adults with myelodysplastic syndromes: the alternative dosing for outpatient treatment (ADOPT) trial. J Clin Oncol 27:3842-3848

17. Jabbour E, Kantarjian H, O'Brien S et al (2013) Retrospective analysis of prognostic factors associated with response and overall survival by baseline marrow blast percentage in patients with myelodysplastic syndromes treated with decitabine. Clin Lymphoma Myeloma Leuk 13:592-596

18. Quintás-Cardama A, Ravandi F, Liu-Dumlao T et al (2012) Epigenetic therapy is associated with similar survival compared with intensive chemotherapy in older patients with newly diagnosed acute myeloid leukemia. Blood 120:4840-4845

19. Lee YG, Kim I, Yoon SS et al (2013) Comparative analysis between azacitidine and decitabine for the treatment of myelodysplastic syndromes. Br J Haematol 161:339-347

20. Lee JH, Choi Y, Kim SD et al (2013) Comparison of 7-day azacitidine and 5-day decitabine for treating myelodysplastic syndrome. Ann Hematol 92:889-897
21. Smith BD, Beach CL, Mahmoud D, Weber L, Henk HJ (2014) Survival and hospitalization among patients with acute myeloid leukemia treated with azacitidine or decitabine in a large managed care population: a real-world, retrospective, claims-based, comparative analysis. Exp Hematol Oncol 3:10

22. Xie M, Jiang Q, Xie Y (2015) Comparison between decitabine and azacitidine for the treatment of myelodysplastic syndrome: a metaanalysis with 1,392 participants. Clin Lymphoma Myeloma Leuk 15:22-28

23. Arber DA, Brunning RD, Orazi A et al (2008) Acute myeloid leukaemia with myelodysplasia-related changes. In: Swerdlow SH, Campo E, Harris NL et al (eds) WHO classification of tumours of haematopoietic and lymphoid tissues, 4th edn. IARC, Lyon, pp 124-126

24. Dombret H, Seymour JF, Butrym A et al (2015) International phase 3 study of azacitidine vs conventional care regimens in older patients with newly diagnosed AML with $>30 \%$ blasts. Blood 126 : 291-299

25. Shen L, Kantarjian H, Guo Y et al (2010) DNA methylation predicts survival and response to therapy in patients with myelodysplastic syndromes. J Clin Oncol 28:605-613

26. Blum W, Garzon R, Klisovic RB et al (2010) Clinical response and miR-29b predictive significance in older AML patients treated with a 10-day schedule of decitabine. Proc Natl Acad Sci U S A 107: 7473-7478

27. Metzeler KH, Walker A, Geyer S et al (2012) DNMT3A mutations and response to the hypomethylating agent decitabine in acute myeloid leukemia. Leukemia 26:11061107

28. Lübbert M, Rüter BH, Claus R et al (2012) A multicenter phase II trial of decitabine as first-line treatment for older patients with acute myeloid leukemia judged unfit for induction chemotherapy. Haematologica 97:393-401

29. Wang H, Fan R, Wang XQ et al (2013) Methylation of Wnt antagonist genes: a useful prognostic marker for myelodysplastic syndrome. Ann Hematol 92:199-209

30. Yi JH, Huh J, Kim HJ et al (2013) Genome-wide single-nucleotide polymorphism array-based karyotyping in myelodysplastic syndrome and chronic myelomonocytic leukemia and its impact on treatment outcomes following decitabine treatment. Ann Hematol 92:459-469

31. Yun H, Damm F, Yap D et al (2014) Impact of MLL5 expression on decitabine efficacy and DNA methylation in acute myeloid leukemia. Haematologica 99:1456-1464

32. Bejar R, Lord A, Stevenson K et al (2014) TET2 mutations predict response to hypomethylating agents in myelodysplastic syndrome patients. Blood 124:2705-2712

33. Momparler RL, Côté S, Eliopoulos N (1997) Pharmacological approach for optimization of the dose schedule of 5-aza-2'deoxycytidine (decitabine) for the therapy of leukemia. Leukemia 11:175-180

34. Cabrero M, Jabbour E, Ravandi F et al (2015) Discontinuation of hypomethylating agent therapy in patients with myelodysplastic syndromes or acute myelogenous leukemia in complete remission or partial response: retrospective analysis of survival after long-term follow-up. Leuk Res 39:520-524

35. de Lima M, Giralt S, Thall PF et al (2010) Maintenance therapy with low-dose azacitidine after allogeneic hematopoietic stem cell transplantation for recurrent acute myelogenous leukemia or myelodysplastic syndrome: a dose and schedule finding study. Cancer 116:5420-5431

36. Pusic I, Choi J, Fiala MA et al (2015) Maintenance therapy with decitabine after allogeneic stem cell transplantation for acute myelogenous leukemia and myelodysplastic syndrome. Biol Blood Transplant 21:1761-1769 